\title{
Estudos descritivos e experimentais em contextos aplicados: dados científicos e impacto prático
}

IIma Aparecida Goulart de Souza Britto. Pontifícia Universidade Católica de Goiás

Roberta Maia Marcon. Pontifícia Universidade Católica de Goiás

\section{Resumo}

O presente artigo se propôs apresentar, a partir de levantamento bibliográfico em periódicos, livros e capítulos de livro, dados da produção intelectual docente e discente oriundos do Programa Stricto Sensu em Psicologia da PUC Goiás, entre os anos 2003 e 2019. Os dados dos estudos possibilitaram ilustrar características do modo de fazer ciência em análise do comportamento aplicada, com destaque a seus métodos e princípios, e ao comportamento-alvo sob estudo. Também permitiram discutir o impacto prático desses estudos. Os resultados mostraram uma maior concentração de estudos descritivos e/ou experimentais em periódicos que abarcam diferentes aspectos do comportamento emitidos por pessoas diagnosticadas com algum transtorno mental, assim como por pessoas que não apresentam diagnóstico psiquiátrico, os quais estavam sob controle de variáveis ambientais testadas nas condições experimentais.

Palavras-chave: comportamento problemático; estudos descritivos e experimentais; análise do comportamento aplicada.

\begin{abstract}
Descriptive and experimental studies in applied contexts. This article aims at presenting the intellectual research of professors and students from the Psychology University of PUC Goiás between the years of 2003 and 2019. The findings made possible to exemplify the human behavior analysis science, highlighting its methods and principles, as well as the target behavior under study. The findings also allowed a discussion of the practical impact of these studies. The results showed a greater concentration of descriptive and/or experimental studies in journals covering different behavioral aspects from people diagnosed with some mental disorder, as well as people who did not present any psychiatric diagnosis but were under control of environmental variables tested in experimental conditions.
\end{abstract}

Keywords: problematic behavior; descriptive and experimental studies; applied behavior analysis.

\section{Resumen}

Estudios descriptivos y experimentales en contextos aplicados. El presente artículo se propuso presentar, a partir de levantamiento bibliográfico en periódicos, libros y capítulos de libro, datos de la producción intelectual docente y discente oriundos del Programa Stricto Sensu en Psicología de la PUC Goiás, entre los años 2003 y 2019. Los datos de los estudios que posibilitaron ilustrar características del modo de hacer ciencia en análisis del comportamiento aplicado, con destaque a sus métodos y principios, y al comportamiento objetivo en estudio. También permitieron discutir el impacto práctico de estos estudios. Los resultados mostraron una mayor concentración de estudios descriptivos y/o experimentales en periódicos que abarcan diferentes aspectos del comportamiento emitidos por personas diagnosticadas con algún trastorno mental, así como por personas que no presentan diagnóstico psiquiátrico, los cuales estaban bajo control de variables ambientales probadas en las condiciones experimentales. Palabras clave: comportamiento problemático; estudios descriptivos y experimentales; análisis de comportamiento aplicado. 
O comportamento, para Skinner (1953), é a relação entre os eventos ambientais e as ações do organismo; por ser um processo e não uma coisa, o comportamento é uma matéria difícil e complexa o que exige técnicas e energia dos cientistas. Sidman (2013) esclarece que o estudo do comportamento é importante pelo seu próprio direito, e as leis da natureza se aplicam diretamente ao que se faz; estas leis incluem variáveis localizadas no sistema nervoso, nos genes, na cultura, ou em qualquer lugar; essas variáveis exercem seus efeitos sobre o comportamento por meio de leis que descrevem o próprio comportamento. Skinner (1956, 1974) argumenta que o comportamento existe somente quando está sendo executado; essa execução requer um sistema biológico que lhe dá sustentação.

Miltenberger e Weil (2013) sugerem que observações e medidas de comportamentos podem assumir muitas formas e envolver uma variedade de métodos qualquer que seja o ambiente. Para tanto, utiliza-se métodos para identificar as relações funcionais entre ambiente-comportamento, de modo que as alterações dessas relações possam resultar em mudanças comportamentais que sejam baseadas em evidências e alcancem impacto social (Baer, Wolf, \& Risley, 1968; Miltenberger, 2012).

Importante ressaltar que os métodos da ciência têm tido um enorme sucesso onde quer que tenham sido aplicados, sendo que os desafios são o de aplicá-los aos assuntos humanos (Skinner, 1953). Os dados são imprescindíveis ao empreendimento científico, uma vez que estes devem ser baseados em evidências.

No âmbito dos conceitos científicos, Madden (2013) declara que a análise do comportamento é, ao mesmo tempo, um campo de estudo, uma disciplina e uma ciência que possui uma abordagem empírica para compreender e influenciar o comportamento. A análise do comportamento (AC) trata das relações funcionais entre os eventos antecedentes e consequentes que controlam as ações do organismo.

Para compreender esse modo peculiar de investigação científica, cumpre citar o fisiologista francês C. Bernard, considerado o pai da medicina experimental, que articulou em termos claros o que nomeou de "experimentos comparativos" com sujeitos intactos e individuais (Iversen, 2013). Ao usar vários animais para comparação, Bernard (1865/1957) notou que os erros em seus dados provinham de variações entre os infrahumanos e "para remover esta fonte de erro, fui obrigado a fazer toda a experiência no mesmo animal" (p. 183). Atendo-se ao estudo de um animal, Bernard (1865/1957) adotou os princípios baseados em evidências diretas do método indutivo.

Os métodos comportamentais focam a construção de uma ciência indutiva. Uma questão que surge diz respeito a que tipos de procedimentos um pesquisador considera importante em seu campo de estudo, pois questões metodológicas não podem ser separadas de questões conceituais subjacentes (Baron \& Perone, 1998). Os analistas do comportamento estudam as relações entre os eventos ambientais e as ações do organismo; essa prática requer procedimentos que revelará o efeito de uma variável de controle específica sobre uma dada resposta de um organismo, o sujeito único (Iversen, 2013).

O método de pesquisa de sujeito único $(N=1)$ se refere a uma vasta coleção de procedimentos e um pequeno números de sujeitos individuais estudados ao longo de várias condições experimentais (Iversen, 2013). Apesar de o experimento envolver mais de um sujeito, cada sujeito é tratado como uma replicação independente, sendo que o próprio comportamento do organismo permanece como unidade de análise (Baron \& Perone, 1998; Perone \& Hursh, 2013). Além disso, o comportamento é observado repetidamente e condições são impostas até que o comportamento-alvo se mostre estabilizado.

Variáveis estranhas são controladas e os efeitos das variáveis independentes (ou variáveis que o pesquisador manipula) são examinados como eles naturalmente ocorrem dentro e através das condições, nos mesmos organismos e não como construção de médias de desempenho de vários grupos (Baron \& Perone, 1998; Lattal, 2013). Este método tem sido usado para especificar aspectos metodológicos básicos nas ciências comportamentais, medicina e medicina comportamental (Iversen, 2013).

\section{A metodologia na prática: aplicações da ciência do comportamento}

Em meados da década de 1950, os métodos e princípios da AC estenderam-se aos contextos aplicados como os hospitais psiquiátricos. Trata-se do trabaIho pioneiro de Skinner e Lindsley que replicaram os procedimentos operantes em pacientes esquizofrênicos 
institucionalizados no Laboratório de Pesquisa de Comportamento da Faculdade de Medicina de Harvard, no Metropolitan State Hospital em Waltham, Massachusetts (Lattal, 2005; Rutherford, 2003; Skinner, 1956).

No laboratório daquele hospital, foi construído um aparelho no qual os pacientes podiam puxar uma alavanca para obter doces, cigarros, imagens de nus projetadas em telas e outros estímulos disponibilizados em esquemas de intervalo variável (VI, variable-interval) e de razão fixa (FR, fixed-ratio). Os registros produziram taxas de resposta comumente observadas com organismos infrahumanos mantidos naqueles esquemas de reforçamento (Lindsley \& Skinner, 1954; Skinner, Solomon, \& Lindsley, 1954).

Embora haja evidências sugerindo que os comportamentos de delirar (falar frases sem sentido) e alucinar (falar que vê, ouve ou sente na ausência de estímulos) emitidos por pessoas com o diagnóstico de esquizofrenia possam ser induzidos por mecanismos biológicos (Associação Americana de Psiquiatria, 2013/2014), os resultados da pesquisa básica e aplicada indicam que estes comportamentos (como outros comportamentos-problema) podem ser tratados ou modificados. Diante disso, prioriza-se uma intervenção cujo foco é a seleção de métodos que, de outro modo, identifica e/ou testa os efeitos de eventos antecedentes e consequentes controladores do comportamento.

Como forma de exemplificar o controle de eventos ambientais, considere o relato de uma pessoa esquizofrênica, "o diabo não me deixa sorrir" (Britto, Rodrigues, Alves, \& Quinta, 2010; Britto, Rodrigues, Santos, \& Ribeiro, 2006). Na tentativa de identificar a função desta vocalização, perguntar-se-ia, o que ou se algo no ambiente mantém esse tipo de relato, uma vez que a frequência do sorrir poderia diminuir, mas a diminuição não poderia ser atribuída a fatos que envolvessem o diabo (estímulo sobrenatural inobservável). Por outro lado, o relato incomum servia como um evento antecedente que permitiria comportamentos incompatíveis com o sorrir, sendo esses comportamentos mantidos por reforçamento negativo, ou seja, pela eliminação, cessação ou adiamento de consequências aversivas que supostamente seriam liberadas pelo diabo se o sorriso ocorresse. Ademais, esse tipo de relato produz a atenção social como evento consequente fornecido pelo ouvinte, bem como o afetaria ao gerar nele respostas emocionais (Britto, 2012, 2017).

A partir de todo o exposto, o presente artigo apresentará dados oriundos da produção intelectual docente e discente da linha de pesquisa denominada Análise do Comportamento do Programa de Pós-Graduação Stricto Sensu em Psicologia (PSSP) da Pontifícia Universidade Católica de Goiás (PUC Goiás), com o objetivo de oferecer um panorama geral dessa área de conhecimento. Os dados são procedentes de publicações em periódicos, livros e capítulos de livros. A partir desses dados, no presente artigo serão apresentadas características do modo de fazer ciência em AC mais especificamente, da análise do comportamento aplicada, ABA (do inglês, Applied Behavior Analysis), em termos de onde estão sendo realizados os estudos, a variável sob estudo, bem como seus princípios e métodos. Também será feita discussão sobre o impacto prático desse campo de atuação a partir dos resultados das aplicações da ABA.

Tendo em vista estes objetivos, o presente trabalho sinaliza que as aplicações de uma ciência do comportamento não estão vinculadas a uma área específica. Lerman, Iwata e Hanley (2013) esclarecem que enquanto a maioria dos campos aplicados em psicologia se distingue por sua ênfase em uma clientela particular, problema, ou configuração, a ABA se limita apenas aos seus princípios e métodos. Por concentrar-se em qualquer aspecto do comportamento humano e, às vezes, não humano, independentemente de quem o emite ou onde ocorre, a ABA extrapola as fronteiras profissionais usadas para definir a psicologia clínica, educacional, organizacional, assim como as categorias dos manuais diagnósticos tradicionais (Lerman et al., 2013).

A Tabela 1 discrimina os estudos dos discentes do PSSP da PUC Goiás sobre as aplicações da AC com ênfase na variável sob estudo, campo de aplicação, princípio e método utilizados. Os dados que serão apresentados correspondem àqueles publicados em periódicos nacionais: Revista Brasileira de Terapia Comportamental e Cognitiva, RBTCC (Britto et al., 2006; Cardoso, Britto, \& Simonassi, 2016; Nóbrega \& Britto, 2017; Novais \& Britto, 2014; Sousa \& Britto, 2017), Psico-USF (Fernandes \& Britto, 2007); Psicologia: Teoria e Pesquisa (Britto et al., 2010; Miranda \& Britto, 2011); Revista Brasileira de Análise do Comportamento, REBAC (Marcon \& Britto, 2015a). 
Tabela 1. Estudos Descritivos e Experimentais sobre as Aplicações da AC

\begin{tabular}{|c|c|c|c|c|}
\hline Estudo & Variável sob estudo & Ambiente & Método & Objetivo \\
\hline Fernandes e Britto (2005) & $\begin{array}{l}\text { Comportamentos verbais e não-verbais da } \\
\text { díade médico-paciente com HIV }\end{array}$ & Hospital público & Descritivo & $\begin{array}{l}\text { Categorias da relação médico e } \\
\text { paciente com HIV }\end{array}$ \\
\hline Fernandes e Britto (2007) & $\begin{array}{l}\text { Ações médicas (informar, investigar, } \\
\text { explicar) na relação com paciente com HIV }\end{array}$ & Hospital público & Descritivo & $\begin{array}{l}\text { Categorias da relação médico e } \\
\text { paciente com HIV }\end{array}$ \\
\hline Miranda e Britto (2011) & $\begin{array}{l}\text { Comportamentos como (dar tapas, } \\
\text { ameaçar, isolar-se, etc.) de pessoa com } \\
\text { esquizofrenia }\end{array}$ & Hospital psiquiátrico & Experimental & $\begin{array}{l}\text { Reforçamento, extinção, modelagem } \\
\text { e modelação }\end{array}$ \\
\hline Novais e Britto (2013) & $\begin{array}{l}\text { Comportamentos-problema de uma } \\
\text { criança vítima de abuso sexual }\end{array}$ & Ambiente doméstico & Experimental & $\begin{array}{l}\text { Fonte de reforçamento positivo, } \\
\text { negativo e automático + DRA }\end{array}$ \\
\hline Nóbrega e Britto (2017) & $\begin{array}{l}\text { Verbalizações negativas e positivas de } \\
\text { duas pessoas com depressão }\end{array}$ & Clínica particular & Experimental & $\begin{array}{l}\text { Fontes de reforçamento positivo, } \\
\text { negativo e automático + DRA }\end{array}$ \\
\hline Sousa e Britto (2017) & $\begin{array}{l}\text { Delirar e alucinar de uma pessoa com } \\
\text { esquizofrenia de comunidade evangélic }\end{array}$ & Clínica particular & Experimental & $\begin{array}{l}\text { Fontes de reforçamento + } \\
\text { discr. verbal e DRA }\end{array}$ \\
\hline
\end{tabular}

Os dados da Tabela 1 permitem afirmar que as aplicações da AC extrapolam qualquer aspecto do comportamento humano (e. g., respostas verbais como delirar, comportamento supersticioso, birras, etc.) independentemente de quem o emite (criança, pessoa diagnosticada com esquizofrenia, depressão etc., ou pessoas não diagnosticadas com transtorno mental) ou onde ocorrem (hospital público ou psiquiátrico, clínica de psicologia, ambiente doméstico, etc.), limitando-se aos seus princípios (reforçamento, extinção, modelagem, modelação, etc.) e métodos (descritivo ou experimental).

Em se tratando dos métodos de pesquisa - descritivo e experimental - os mesmos são empregados para identificação das funções comportamentais. Cumpre salientar que, ainda que nos estudos (Tabela 1) se utilizem os métodos supracitados, comumente recorre-se a avaliação funcional indireta para identificar eventos comportamentais e ambientais. Nesse sentido, os pesquisadores perguntam uma série de questões sobre o comportamento e eventos ambientais a pais, profissionais de saúde, cônjuges ou a outras pessoas relevantes àquele que emite comportamentos-problema que, por sua vez, possam fornecer dados importantes para avaliação funcional. Um dos instrumentos usados é a Entrevista de Avaliação Funcional desenvolvida por O'Neill et al. (1997), adaptada e publicada por Oliveira e Britto (2011).

A Tabela 2 apresenta o resumo dos métodos usados para identificar as funções dos eventos determinantes do comportamento-alvo, como se segue.

Tabela 2. Métodos para Manipular Comportamentos-Alvo com Base em suas Funções

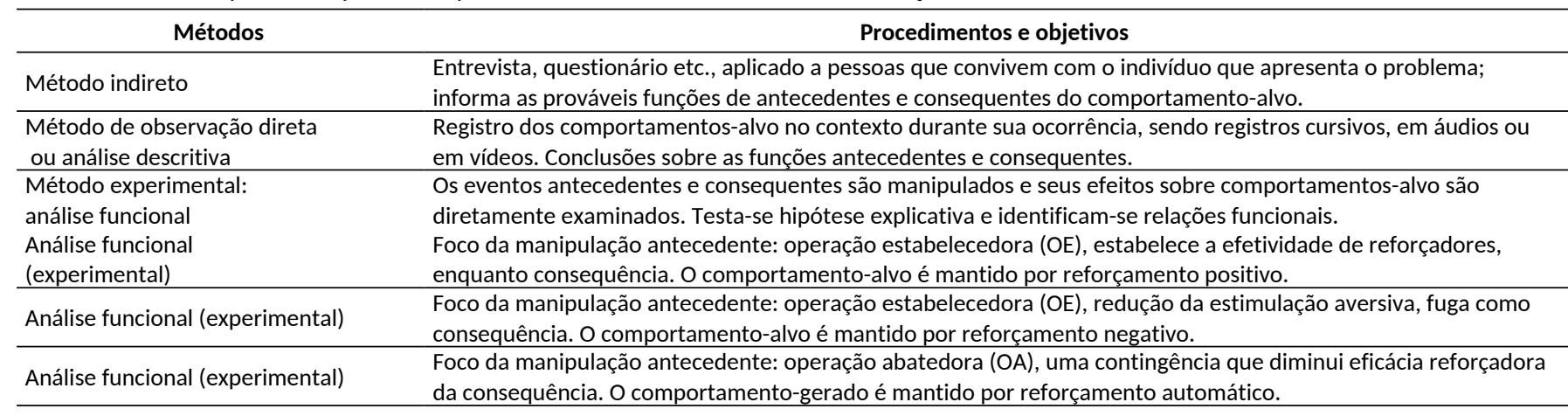


No que diz respeito ao método indireto, com o uso de entrevistas, é relevante discutir alguns aspectos destes dados como em relação aos comportamentos-alvo de pessoas com diagnóstico de esquizofrenia (e. g., vocalizações bizarras, delirantes, confusas ou desconexas), cujas informações são prestadas por familiares e pelos profissionais de saúde. Os dados apontados por Marcon e Britto (2015b) informam, por um lado, que tantos os familiares quanto profissionais de saúde explicam as falas pela cronicidade do quadro de esquizofrenia, ignorando eventos ambientais relevantes; e ainda, que as pessoas viviam fora da realidade e a saída da instituição as prejudicariam (Alves \& Britto, 2019), o que ratifica uma explicação baseada em mecanismo subjacente à pessoa, portanto, em algo que a pessoa portaria, quer seja, a esquizofrenia. Além disso, também apontaram eventos ambientais mantenedores das vocalizações bizarras, uma vez que estas eram consequenciadas com alguns privilégios como evitar atividades domésticas ou obter cigarros, café e adiar banhos fora dos horários estabelecidos pela instituição (Bueno \& Britto, 2013; Sousa \& Britto, 2017).

Em relação ao método descritivo, com o uso de observação direta do comportamento-alvo no ambiente natural (ambiente doméstico, hospital público) é possível obter conclusões válidas sobre as funções dos comportamentos-problema. Para exemplificar, cumpre citar os dados do estudo de Novais e Britto (2013) sobre as funções dos comportamentos-problema de uma criança que fora vítima de abuso sexual. Em situação de observação em ambiente doméstico, a pesquisadora registrou que a criança pede a mãe e a babá para brincar na sala - que acabara de ser arrumada ao que ambas negam sugerindo que ela procurasse outro brinquedo (evento antecedente). Em resposta a criança faz birra, joga-se no chão, grita e chora dizendo "hoje estou triste porque lembrei que o papai colocou o ' $p$ ' em minha ' $p$ '”. A mãe também chora, enquanto abraça e coloca a filha no colo, adiando sua ida para o trabalho (evento consequente).

Quanto aos registros de autogravações, cumpre citar os fornecidos por uma das participantes do estudo de Menezes e Britto (2019) sobre os momentos desagradáveis de sua rotina na agência bancária. Os dados apontaram:

Ele acabou de me dizer que existem muitos querendo a minha vaga aqui no banco e eu quero ver dar conta dessa pressão. Tem muita planilha para fazer aqui que não dá tempo de produzir.
Aqui é a planilândia. O sistema demora atualizar três dias e o superintendente quer a atualização diária, tudo tem que ser na hora dele, nunca espera o tempo do sistema.

Estes dados denotam como a participante se sentia pressionada pelo superintendente a cumprir uma exigência na empresa bancária sob a ameaça de perder o emprego.

Finalmente, a respeito do método experimental, é importante destacar, de início, que as variáveis manipuladas pelo pesquisador são identificadas com base nas variáveis contextuais aferidas durante passos preliminares, em que se empregam os métodos de avaliação indireta e descritiva (Tabela 2). Os eventos ambientais antecedentes são manipulados ao longo da exposição do participante à contingência em vigor e, igualmente, os eventos ambientais consequentes reforçadores do comportamento-alvo.

Em se tratando da análise funcional (experimental) esta abarca condições experimentais (atenção, demanda, sozinho, controle) para examinar diretamente o controle do reforçamento positivo, negativo e automático (que independe de mediação social) sobre o comportamento-problema (Iwata, Dorsey, Slifer, Bauman, \& Richman, 1982/1994). Ademais, as variáveis são manipuladas na presença de operações motivadoras OM (estabelecedora, OE e abolidora, AO), portanto, de variáveis antecedentes que influenciam o comportamento. Para justificar a importância do uso desta metodologia, Marcon e Britto (2015b) esclarecem que esta se dá em função do procedimento de reunir os estímulos discriminativos $\left(\mathrm{S}^{\mathrm{D}}\right)$, as $\mathrm{OM}$ e os potenciais reforçadores, manipulados de modo controlado para elucidar e isolar os efeitos de potenciais fontes de reforçamento.

Para exemplificar as manipulações da análise funcional (experimental) no contexto dos trabalhos apresentados ao PSSP da PUC Goiás, Marcon e Britto (2015b) investigaram o controle exercido pelos eventos antecedentes, o responder pelo $S^{D}$ ou pela OM, e também pelos eventos reforçadores consequentes sobre as respostas verbais de pessoas com diagnóstico de esquizofrenia, sendo manipuladas as condições na presença de $\mathrm{OM}$ e na presença de reforçamento positivo e negativo.

Os dados apontaram que as vocalizações bizarras dos participantes foram emitidas com alta frequência nas condições atenção e fuga de demanda, sendo evidenciado o controle do responder pela OM e pelo reforçamento. $\mathrm{Na}$ condição sozinho, as vocalizações bizarras foram

Estudos de Psicologia, 24(2), abril a junho de 2019, 204-214 
suprimidas, sendo evidenciado o controle sobre o responder quando na presença de operação abolidora e ausência de operação de reforçamento. Na condição controle, foi possível deduzir o efeito da OM de aumentar a eficácia do estímulo reforçador. Contudo, as vocalizações bizarras não foram emitidas haja vista que o estímulo reforçador foi disponibilizado independentemente da ocorrência das mesmas estando ausente uma operação de reforçamento.

Ainda em relação à condição sozinho, destaca-se o estudo de Sousa e Britto (2019) com drogaditos, que produziram altas frequências de estimulações sensoriais. O ponto essencial dos dados das manipulações das condições apontou que as relações entre antecedentes e consequentes são diretamente verificadas, o que justifica a importância de se analisar, com igual relevância, o controle destes eventos sobre o responder em manipulações experimentais no contexto aplicado (Marcon \& Britto, 2015b).

Tendo como base os métodos indireto, direto e experimental, Menezes e Britto (2019) mostraram como um ambiente hierarquizado de empresas prestadoras de serviços bancários, onde predominava excessos de trabaIhos, cobranças e pressões, aumentaria a efetividade da atenção social (OE) e a probabilidade do cumprimento de metas pelas gerentes como forma de reforçamento positivo, sendo manipulado na condição de atenção. Para a condição de demanda, a apresentação de uma tarefa com uma figura caricata (chefe autoritário, OE) estabeleceria a eficácia reforçadora da retirada da tarefa como forma de reforçamento negativo. Na condição sozinho presença de ambiente não social (OA) e ausência do reforço. $\mathrm{Na}$ condição controle, a presença de revistas, jogos de palavras cruzadas, comestíveis e de bolas antiestresse funcionariam como reforçadores. Os dados da aplicação e replicação apontaram que nas condições de atenção e demanda os relatos das gerentes em relação aos problemas comportamentais foram mais frequentes, quando comparados às demais condições.

Dentre os dados aferidos da relação empresarial destacaram-se (tentativa de ignorar as cobranças, problemas com supervisores, descontentamento dos colegas, prejuízos das relações familiares, agir contra os próprios valores); estados emocionais negativos (e.g., insônias, autopunições como culpar-se o tempo todo, sentir-se incapaz e injustiçada); respostas fisiológicas (e.g., desconforto torácico, mal estar estomacal, aceleração dos batimentos cardíacos, despersonalização, tensões musculares); além de problemas de ordem física (e.g., enxaqueca, gastrite, dores corporais, queda de cabelos e de imunidade).
Do mesmo modo, Vieira e Britto (2019) evidenciaram o quanto os ambientes familiares e hospitalares disponibilizam consequências diferenciais para as respostas de dor, enquanto ignoram as de enfrentamento. Durante a observação direta e indireta, constatou-se um ambiente social onde predominava escassez de interações sociais e de atividades laborativas (OE), o que aumentava a efetividade reforçadora da atenção social e o expressar dor como forma de obter reforçador positivo, sendo manipulado na condição de atenção. Permanecer deitado na maior parte do dia (OE) estabeleceria a efetividade reforçadora da retirada de uma demanda e a resposta de relatar ou expressar dor como forma de fuga das atividades. $\mathrm{Na}$ condição sozinho, um ambiente não social (AO) na ausência de reforço reduziria a probabilidade de emissão do comportamento de expressar dor. $\mathrm{Na}$ condição controle, a inexistência de atividades laborativas (OE) estabeleceria a efetividade reforçadora da interação com alguns reforçadores. Os resultados mostraram que os relatos de dor aumentaram (atenção e demanda) e reduziram (sozinho e controle). Ressalta-se que um padrão comportamental evitativo diante de atividades recomendadas começa a ser selecionado, o que pode levar a perda de reforçadores como os monetários e os sociais. Os dados apontam que o participante não se engaja nas atividades prescritas, o que leva a supor que ele foge e esquiva de próprias sensações de dor, enquanto evento interno que adquire funções discriminativas, permitindo-se ser avaliado como pessoa frágil e incapacitado.

No âmbito dos conceitos científicos já sinalizados para a condução dos estudos, em busca dos eventos antecedentes e consequentes ao comportamento-alvo, torna-se imprescindível analisar a função deste comportamento na tentativa de identificar a contingência mais relevante para a sua ocorrência. Observar os eventos que lhe deram origem, eliminar a fonte de reforço que o controlava, bem como programar eventos específicos, que formariam a base para as manipulações da análise funcional. Deve-se em seguida, programar as intervenções para modificar o comportamento-alvo. Comumente, fez-se uso do reforçamento diferencial de comportamento alternativo (DRA) e a discriminação verbal, dentre tantos outros (ver Tabela 1). Cumpre salientar que todos os procedimentos foram controlados por delineamentos experimentais $N=1$.

A Tabela 3 apresenta dados dos estudos dos discentes do PSSP da PUC Goiás sobre as aplicações da $A C$ que foram publicados no formato de livros, assim também como de estudos submetidos para publicação. 
Estudos descritivos e experimentais em contextos aplicados: dados científicos e impacto prático

Tabela 3. Objetivos, Autores, Editora e Resultados das Aplicações da AC em Formatos de Livros

\begin{tabular}{|c|c|c|}
\hline Objetivos do estudo & Autores e editora & Resultados \\
\hline $\begin{array}{l}\text { Avaliar e tratar os excessos e os déficits } \\
\text { comportamentais de uma pessoa com Síndrome de } \\
\text { Down com o uso das estratégias da AC. }\end{array}$ & $\begin{array}{c}\text { Oliveira e Britto (2011) } \\
\text { ESETEC }\end{array}$ & $\begin{array}{l}\text { Aumento dos comportamentos desejáveis e diminuição } \\
\text { dos indesejáveis além de instalar novas habilidades } \\
\text { comportamentais. }\end{array}$ \\
\hline $\begin{array}{l}\text { Avaliar os efeitos dos eventos antecedentes e } \\
\text { consequentes no controle das respostas verbais de } \\
\text { esquizofrênicos, além de intervir nas classes verbais e } \\
\text { treinar os profissionais na instituição. }\end{array}$ & $\begin{array}{c}\text { Bueno e Britto (2013) } \\
\text { JURUÁ }\end{array}$ & $\begin{array}{l}\text { Comportamentos mantidos pelas fontes de } \\
\text { reforçamento positivo (obter atenção, tangíveis e } \\
\text { privilégios) e negativo (escapar de demandas, adiarem } \\
\text { banhos). O DRA diminuiu as bizarrices. }\end{array}$ \\
\hline $\begin{array}{l}\text { Identificar o controle do responder de esquizofrênicos } \\
\text { pelo estímulo discriminativo, pelas operações } \\
\text { estabelecedoras (OE) e abolidoras (AO), bem como } \\
\text { a função de reforçadores consequentes sobre as } \\
\text { respostas. }\end{array}$ & $\begin{array}{c}\text { Marcon e Britto (2015b) } \\
\text { CRV }\end{array}$ & $\begin{array}{l}\text { Identificou-se o controle pelas operações motivadoras } \\
\text { (OE e AO) que aumentava ou reduzia a frequência de } \\
\text { comportamentos dependendo do tipo de OE e OA que } \\
\text { alteravam os efeitos dos reforçadores. }\end{array}$ \\
\hline $\begin{array}{l}\text { Avaliar a emissão de comportamento supersticioso } \\
\text { de pessoas da comunidade quando submetidos a três } \\
\text { condições: FT } 20 \text { seg., FI 30seg. e de extinção. }\end{array}$ & $\begin{array}{c}\text { Cardoso e Britto (2018) } \\
\text { CRV }\end{array}$ & $\begin{array}{l}\text { Nenhum dos participantes discriminou as condições } \\
\text { vigentes; responderam de modo estereotipado } \\
\text { compatível ao comportamento supersticioso. }\end{array}$ \\
\hline $\begin{array}{l}\text { Avaliar e tratar os comportamentos de pessoas que se } \\
\text { encontravam internadas em instituição comunitária } \\
\text { religiosa para se livrarem das drogas. }\end{array}$ & $\begin{array}{c}\text { Sousa e Britto (2019) } \\
\text { CRV }\end{array}$ & $\begin{array}{l}\text { A avaliação determinou o planejamento do tratamento } \\
\text { com programa de autogerenciamento que produziu } \\
\text { mudanças relevantes nos comportamentos drogaditos. }\end{array}$ \\
\hline $\begin{array}{l}\text { Avaliar e tratar as respostas bizarras emitidas por } \\
\text { esquizofrênicas com o uso da análise funcional. } \\
\text { Tratamento: discriminação verbal e DRA. }\end{array}$ & $\begin{array}{l}\text { Alves e Britto (2019) } \\
\text { CRV }\end{array}$ & $\begin{array}{l}\text { Aumento de bizarrices durante condições de } \\
\text { atenção e demanda. Durante o tratamento, DRA e a } \\
\text { discriminação verbal controlaram as respostas bizarras. }\end{array}$ \\
\hline
\end{tabular}

Os dados da Tabela 3 elucidam diferentes aspectos do comportamento humano (excessos e os déficits comportamentais de uma pessoa com Síndrome de Down, respostas verbais de pessoas com esquizofrenia, comportamentos de drogaditos em instituição comunitária religiosa, comportamento supersticioso de pessoas da comunidade, comportamentos de gerentes em empresa bancária, respostas de dor em ambiente hospitalar), o que apontam que as aplicações da AC extrapolam as fronteiras profissionais usadas para definir a psicologia clínica, educacional, organizacional, assim como as categorias dos manuais diagnósticos tradicionais. Também apresentam resultados oriundos da pesquisa básica e aplicada com ênfase no efeito dos eventos antecedentes e consequentes controladores do comportamento-alvo, indicando, pois, que estes comportamentos podem ser tratados ou modificados.

Ademais, ilustra dados da pesquisa básica, por exemplo, Cardoso e Britto (2018) estudaram os comportamentos de seis participantes que foram submetidos à condição com os parâmetros da proximidade acidental de uma resposta e um reforçador: tempo fixo (fixed time, FT) de 20 segundos; uma de intervalo fixo (interval fixed, FI) de 30 segundos. Também foi programada uma condição de extinção (EXT). Os participantes produziram estereotipias variadas que podem ser interpretadas como comportamento supersticioso. Some-se a isto, que o comportamento supersticioso pode contribuir para a generalidade de fenômenos descritos como ilusão de controle, conceito da psicologia social experimental.

A Tabela 4 aponta os estudos de discentes e docentes do PSSP cujas temáticas variam, desde observações do comportamento no contexto de uma clínica escola de psicologia a conteúdos teóricos diversificados envolvendo transtorno de pânico, esquizofrenia, tricotilomania, dependências de substâncias, emoções, comportamento sexual, ansiedade, os quais foram publicados em diferentes periódicos. 
Tabela 4. Autores, Periódicos e Objetivos de Estudos com Conteúdos Variáveis

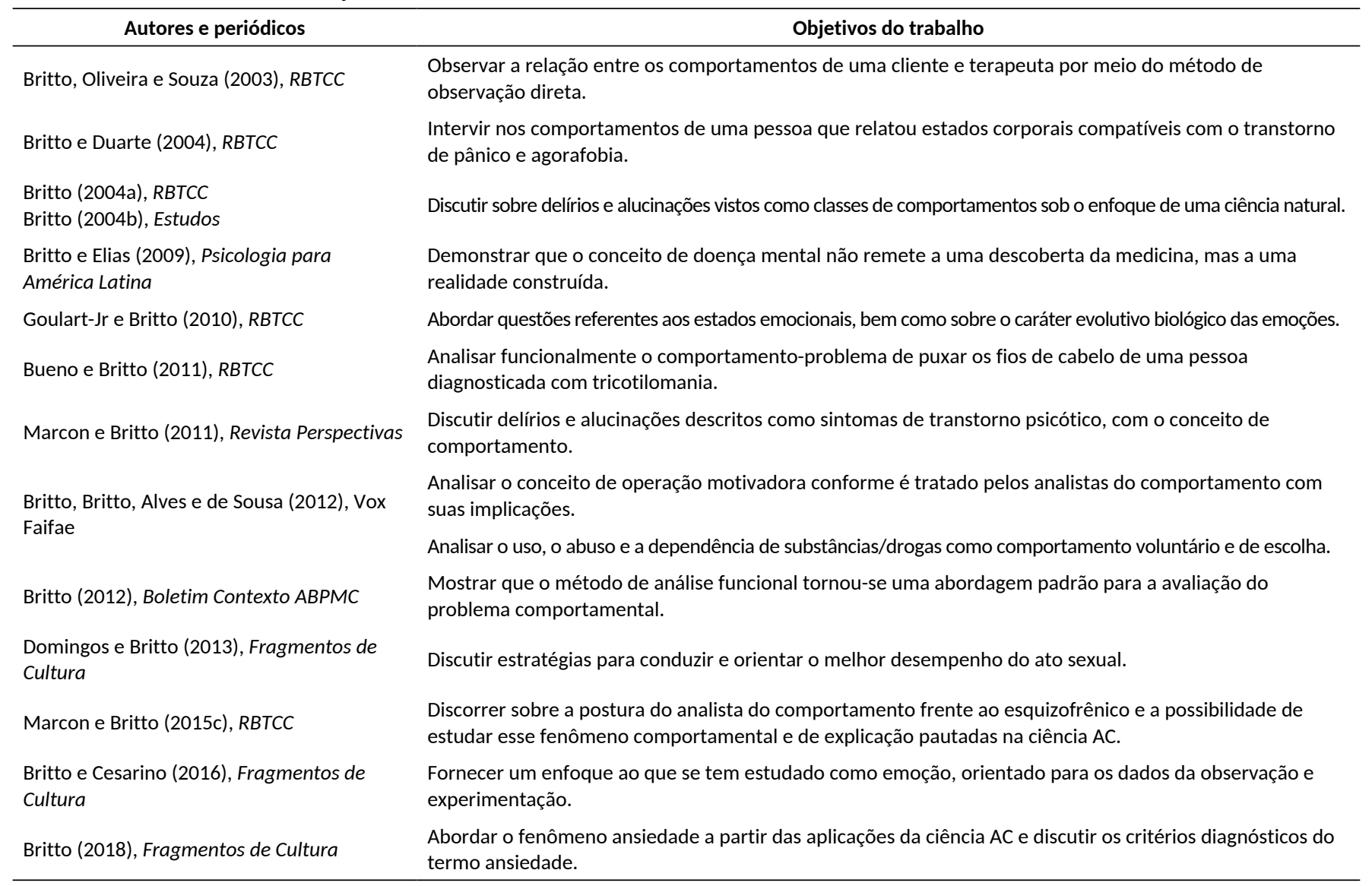

Na maior parte dos estudos da Tabela 4, privilegiaram-se considerações sobre a análise do comportamento e os comportamentos-problema - comumente diagnosticados sob o rótulo de esquizofrenia, ansiedade, tricotilomania, dependência de substância - em contraposição as concepções e explicações destes pela visão tradicional psiquiátrica. As classificações psiquiátricas, ainda que detenham significado social e institucional, podem ser questionadas, uma vez que a confiabilidade e a validade de suas categorias diagnósticas não são testadas por meio de exames laboratoriais (Britto, 2004a).

Uma maneira resumida de apresentar dados gerais da produção analítico-comportamental discente e docente oriundos do PSSP da PUC Goiás será apresentada na Figura 1.

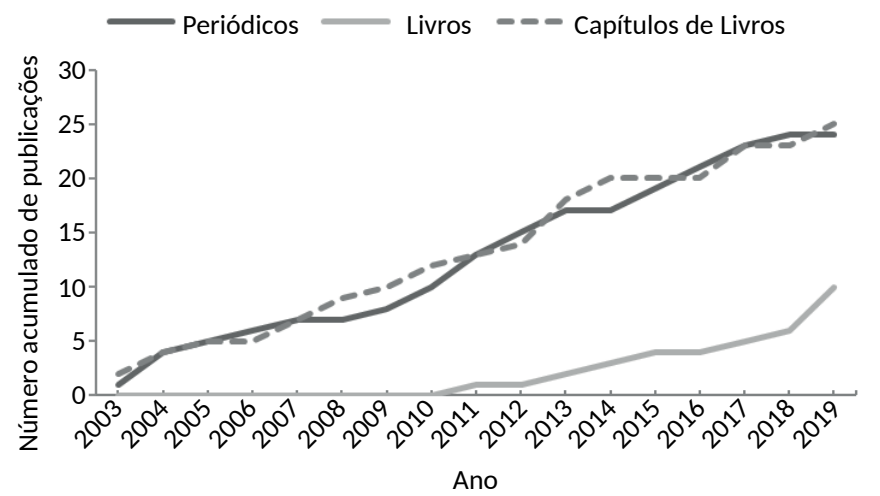

Figura 1. Anos e Publicações em Periódicos, Livros e Capítulos de Livros.

A Figura 1 apresenta três curvas acumuladas: (1) número de publicações em AC em periódicos, pautado em estudos empíricos e/ou descritivos, estudos 
teóricos e estudos de caso, (2) número de publicações em AC em livros, relativo a estudos empíricos e (3) número de publicações em AC em capítulos de livros, referente a estudos empíricos e/ou descritivos, estudos teóricos e estudos de caso.

Observa-se uma maior frequência de publicações em capítulos de livros (25 publicações de 2003 a 2019), podendo ser notado quatro publicações ao ano. As publicações em periódicos seguem como segundas mais frequentes (24 publicações no referido intervalo de tempo), podendo ser observada três publicações ao ano. Por fim, as publicações em livros (10 publicações) com quatro publicações como maior número de publicações em um mesmo ano. Observa-se que a maior concentração de estudos empíricos e/ou descritivos (12 estudos) está publicada em periódicos, enquanto que a maior concentração de publicação de estudos teóricos (19) foi em capítulos de livro. Já os estudos de caso também foram publicados em sua maioria (2) em capítulos de livro.

Do exposto, poder-se-ia concluir que o presente levantamento bibliográfico em periódicos, livros e capítulos de livro forneceram dados acerca da produção intelectual docente e discente oriundos do PSSP da PUC Goiás. A partir desses dados foi possível apresentar características do modo de fazer ciência em ABA, cujas pesquisas se caracterizam por medidas do comportamento em diferentes condições e em poucos sujeitos. Para além dos critérios científicos apresentados, cumpre destacar o impacto prático desses estudos, oriundo das mudanças no comportamento para o próprio participante, para pessoas que lhe são importantes e para a sociedade em geral. Nos estudos supracitados o comportamento-alvo é de relevância social e os dados oriundos das aplicações asseguram a eficácia dos resultados na modificação do comportamento, especialmente, se comparados aos resultados produzidos pelo tratamento convencional.

Como forma de exemplificar sua relevância, destaca-se, por fim, dados do estudo de Vieira e Britto (2019). Evidenciou-se que apesar da grande quantidade de medicamentos consumidos por participantes com dor crônica, estes não descreveram melhoras de alívio da dor e ainda problemas emocionais (e.g., insônia, ansiedade, depressão) em função da persistência de dores, o que, por sua vez, favorece para a administração de mais medicamentos por parte da equipe médica, o que acaba por produzir uma espécie de círculo vicioso que pode ser difícil quebrar.

\section{Referências}

Alves, J. C., \& Britto, I. A. G. S. (2019). Sobre a esquizofrenia. Curitiba: CRV.

Associação Americana de Psiquiatria. (2014). Manual diagnóstico e estatístico de transtornos mentais ( $5^{\mathrm{a}}$ edição, M. I. C. Nascimento, P. H. Machado, R. M. Garcez, R. Pizzato, \& S. M. M. Rosa, Trads.). Porto Alegre: ARTMED. (Obra original publicada em 2013)

Baer, D. M., Wolf, M. M., \& Risley, T. R. (1968). Some current dimensions of applied behavior analysis. Journal of Applied Behavior Analysis, 1, 91-97. doi: 10.1901\%2Fjaba.1968.1-91

Baron, A., \& Perone, M. (1998). Experimental designs and analysis in the laboratory study of human operant behavior. In K. A. Lattal \& M. Perone (Orgs.), Handbook of research methods in human operant behavior (pp. 45-91). Plenum Press, Nova lorque: Springer.

Bernard, C. (1957). An introduction to the study of experimental medicine. Nova lorque: Dover. (Obra original publicada em 1865)

Britto, I. A. G. S. (2004a). Sobre delírios e alucinações. Revista Brasileira de Terapia Comportamental e Cognitiva, 6(1), 61-71. Recuperado de http://pepsic.bvsalud.org/scielo. php?script=sci_arttext\&pid=S1517-55452004000100007\&lng= pt\&tlng=pt

Britto, I. A. G. S. (2004b). As implicações práticas do conceito de doença mental. Estudos, 31(spe), 157-172. Recuperado de http://professor. pucgoias.edu.br/SiteDocente/admin/arquivosUpload/1711/material/ Implica\%C3\%A7\%C3\%B5es\%20pr\%C3\%A1ticas\%20do\%20 conceito\%20de\%20doen\%C3\%A7a\%20mental.pdf

Britto, I. A. G.S. (2012). Psicopatologia e análise do comportamento: algumas reflexões. Boletim Contexto, 37(2), 55-76. Recuperado de http://abpmc. org.br/arquivos/publicacoes/1405369626aca352164b09.pdf

Britto, I. A. G. S. (2017). Abordagem funcional para a esquizofrenia. Curitiba: Appris.

Britto, I. A. G. S. (2018). Abordagem analítico-comportamental para o estudo do fenômeno ansiedade e suas implicações. Fragmentos de Cultura, 28(spe), 90-100. doi: 10.18224/frag.v28i2.5480

Britto, I. A. G. S, Britto, A. L. G. S., Alves, J. C., \& Sousa, N. R. (2012) Sobre o comportamento de consumir e depender de substâncias. Vox Faifae: Revista de Teologia, 4(1), 1-14. Recuperado de http://www.faifa.edu.br/revista/index.php/voxfaifae/article/view/56

Britto, I. A. G. S., \& Cesarino, A. M. (2016). Análise do comportamento e o fenômeno emocional. Fragmentos de Cultura, 26(2), 187-19. doi: 10.18224/frag.v26i2.4893

Britto, I. A. G.S., \& Duarte, A. M. (2004). Transtorno de pânico e agorafobia: um estudo de caso. Revista Brasileira de Terapia Comportamental e Cognitiva, 6(2), 165-172. Recuperado de http://pepsic.bvsalud.org/ scielo.php?script=sci_arttext\&pid=S1517-55452004000200003\&lng= pt\&tlng $=\mathrm{pt}$

Britto, I. A. G.S., \& Elias, P.V.O. (2009). Análise comportamental das emoções. Psicologia para América Latina, 16. Recuperado de http://pepsic. bvsalud.org/scielo.php?script=sci_arttext\&pid=\$1870-350X20090 00100004\&lng=pt\&tlng=pt

Britto, I. A. G. S., Oliveira, J. A., \& Sousa, L. F. D. (2003). A relação terapêutica evidenciada através do método de observação direta. Revista Brasileira de Terapia Comportamental e Cognitiva, 5(2), 139-149. doi: 10.31505/rbtcc.v5i2.77

Britto, I. A. G. S., Rodrigues, I. S., Alves, S. L., \& Quinta, T. L. S. S. (2010). Análise funcional de comportamentos verbais inapropriados de um esquizofrênico. Psicologia: Teoria e Pesquisa, 26(1), 139-144 doi: 10.1590/S0102-37722010000100016 
Britto, I. A. G.S., Rodrigues, M. C. A., Santos, D.C. O., \& Ribeiro, M. A.(2006). Reforçamento diferencial de comportamentos verbais alternativos de um esquizofrênico. Revista Brasileira de Terapia Comportamental e Cognitiva, 8(1), 73-84. Recuperado de http://pepsic.bvsalud.org/scielo. php?script=sci_arttext\&pid=S1517-55452006000100007\&lng=pt\& tlng=pt

Bueno, G. N., \& Britto, I. A. G. S. (2011). Uma abordagem funcional para os comportamentos delirar e alucinar. Revista Brasileira de Terapia Comportamental e Cognitiva, 8(3), 4-15. Recuperado de http://pepsic.bvsalud.org/scielo. php?script=sci_arttext\&pid=S1517-55452011000300002\&lng= pt\&tlng $=p t$

Bueno, G. N., \& Britto, I. A. G. S. (2013). A esquizofrenia de acordo com a abordagem comportamental. Curitiba: Juruá.

Cardoso, L. A., \& Britto, I. A. G. S. (2018). O estudo do comportamento supersticioso. Curitiba: CRV.

Cardoso, L. A., Britto, I. A. G. S., \& Simonassi, L. E. (2016). Comportamento supersticioso em estudantes universitários. Revista Brasileira de Terapia Comportamental e Cognitiva, 18(1), 31-44. doi: 10.31505/ rbtcc.v18i1.829

Domingos, V. G. M., \& Britto, I. A. G. S. (2013). Disfunção sexual masculina: algumas implicações. Fragmentos de Cultura, 23(4), 579-586. doi: 10.18224/frag.v23i4.2988

Fernandes, C. R. D., \& Britto, I. A. G. S. (2005). Atuação médica frente ao paciente portador de HIV no contexto ambulatorial. Revista Brasileira de Terapia Comportamental e Cognitiva, 7(2), 205-218. Recuperado de http://pepsic.bvsalud.org/scielo. php?script=sci_arttext\&pid=S1517-55452005000200006\&lng= pt\&tlng $=\mathrm{pt}$

Fernandes, C. R. D., \& Britto, I. A. G. S. (2007). Ação médica no contexto ambulatorial com o portador de HIV. Psico-USF, 12(2), 309-318. doi: 10.1590/S1413-82712007000200019

Goulart-Jr, R. M., \& Britto, I. A. G. S (2010). Intervenção analíticocomportamental em tricotilomania. Revista Brasileira de Terapia Comportamental e Cognitiva, 12(1/2), 224-237. doi: 10.31505/ rbtcc.v12i $1 / 2.423$

Iversen, I. H. (2013). Single-case research methods: An overview. In G. J. Madden, W. V. Dube, T. D. Hackenberg, G. P. Hanley, \& K. A. Lattal (Orgs.), Handbook of behavior analysis (Vol. 1, pp. 3-32). Washington, DC: APA Handbook in Psychology.

Iwata, B. A., Dorsey, M. F., Slifer, K. J., Bauman, K. E., \& Richman, G. S. (1994). Toward a functional analysis of self-injury. Journal of Applied Behavior Analysis, 27(2), 197-209. doi: 10.1901/jaba.1994.27-197. (Reedição de Analysis and Intervention in Developmental Disabilities, 2(1), 3-20 (1982). doi: 10.1016/0270-4684(82)90003-9)

Lattal, K. A. (2005). Ciência, tecnologia e análise do comportamento. In J. Abreu-Rodrigues \& M. R. Ribeiro (Orgs.), Análise do comportamento: pesquisa, teoria e aplicação (pp. 15-26). Porto Alegre: Artmed.

Lattal, K. A. (2013). The fives pillars of the experimental analysis behavior. In G. J. Madden, W. V. Dube, T. D. Hackenberg, G. P. Hanley, \& K. A. Lattal (Orgs.), Handbook of behavior analysis (Vol. 1, pp. 33-64). Washington: APA Handbook in Psychology.

Lerman, D. C., Iwata, B. A., \& Hanley, G. P. (2013). Applied behavior analysis. In G. J Madden, W. V. Dube, T. D. Hackenberg, G. P. Hanley, \& K. A. Lattal (Orgs.), Handbook of behavior analysis (Vol. 1, pp. 81-104). Washington: APA Handbook in Psychology.

Lindsley, O. R., \& Skinner, B. F. (1954). A method for the experimental analysis of the behavior of psychotic patients. American Psychologist, 9, 419-420.
Madden, G. J. (2013). Introduction. In G. J. Madden, W. V. Dube, T. D. Hackenberg, G. P. Hanley, \& K. A. Lattal (Orgs.), Handbook of behavior analysis (Vol. 1, pp. 21-24). Washington: APA Handbook in Psychology.

Marcon, R. M., \& Britto, I. A. G. S. (2011). Operações motivadoras e atenção social: eventos relevantes para comportamentos-problema de esquizofrênicos. Revista Perspectivas em Análise do Comportamento, 2(2), 192-202. Recuperado de http://pepsic.bvsalud.org/scielo. php?script=sci_arttext\&pid=S2177-35482011000200005\&Ing= pt\&tlng=pt

Marcon, R. M., \& Britto, I. A. G. S. (2015a). Análise funcional de falas inapropriadas em uma pessoa com diagnóstico de esquizofrenia. Revista Brasileira de Análise do Comportamento, 11(1), 53-60. doi: 10.18542/rebac.v11i1.3774

Marcon, R. M., \& Britto, I. A. G. S. (2015b). O controle pelos antecedentes e consequentes nas respostas verbais de pessoas com diagnóstico de esquizofrenia. Curitiba: CRV.

Marcon, R. M., \& Britto, I. A. G.S. (2015c). O estudo do comportamento do psicótico: contribuições analítico-comportamentais. Revista Brasileira de Terapia Comportamental e Cognitiva, 17(1), 23-34. doi: 10.31505/ rbtcc.v17i1.733

Menezes, N. K. B., \& Britto, I. A. G. S. (2019). Avaliação funcional dos comportamentos de gerentes em empresas prestadoras de serviços bancários (Tese de Doutorado, Pontifícia Universidade Católica de Goiás, Goiás). Recuperado de http://tede2.pucgoias.edu.br:8080/ handle/tede/4236

Miltenberger, R. G. (2012). Behavior modification: Principles and procedures. (5 Edição). Belmont: Wadsworth.

Miltenberger, R., G., \& Weil, T. M. (2013). Observation and measurement in behavior analysis. In G. J. Madden, W. V. Dube, T. D. Hackenberg, G. P. Hanley, \& K. A. Lattal (Orgs.), Handbook of behavior analysis (Vol. 1, pp. 127-150). Washington: APA Handbook in Psychology.

Miranda, E., \& Britto, I. A. G. S. (2011). Aplicação dos princípios analítico-comportamentais para alterar o comportamento de uma esquizofrênica. Psicologia: Teoria e Pesquisa, 37(3), 327-336. doi: 10.1590/S0102-37722011000300008

Moura, L. F., \& Britto, I. A. G. S. (2017). Esquizofrenia: um estudo experimental sobre delírios e alucinações. Saarbrücken: Novas Edições Acadêmica.

Nóbrega, L. G., \& Britto, I. A. G. S (2017). Avaliação e tratamento de comportamentos problemas de duas pessoas com o diagnóstico de depressão. Revista Brasileira de Terapia Comportamental e Cognitiva, 19(1), 128-145. doi: 10.31505/rbtcc.v19i1.956

Novais, M. R., \& Britto, I. A. G. S. (2013). Comportamentos-problema de uma criança vítima de abuso sexual. Revista Brasileira de Terapia Comportamental e Cognitiva, 15(1), 4-19. doi: 10.31505/rbtcc.v15i1.563

Novais, M. R., \& Britto, I. A. G.S (2014). Modificação de comportamentos: uma criança vítima de abuso sexual. Saarbrücken: Novas Edições Acadêmica.

O'Neill, R. E., Horner, R. H., Albin, R. W., Sprague, J. R., Storey, K., \& Newton, J. S. (1997). Functional assessment and program development for problem behavior: A practical handbook. Pacific Grove: Brooks/Cole.

Oliveira, I. J. S., \& Britto, I. A. G. S. (2011). Síndrome de Down: modificando comportamentos. Santo André: ESETec Editores Associados.

Perone, M., \& Hursh, D. E. (2013). Single-case experimental designs. In G. J. Madden, W. V. Dube, T. D. Hackenberg, G. P. Hanley, \& K. A. Lattal (Orgs.), Handbook of behavioranalysis (Vol.1, pp. 107-126). Washington: APA Handbook in Psychology. 
Estudos descritivos e experimentais em contextos aplicados: dados científicos e impacto prático

Rutherford, A. (2003). Skinner boxes for psychotics: Operant conditioning at Metropolitan State Hospital. The Behavior Analyst, 26(2), 267-279. doi: $10.1007 \% 2 F b f 03392081$

Sidman, M. (2013). Foreword. In G. J. Madden, W. V. Dube, T. D. Hackenberg, G. P. Hanley, \& K. A. Lattal (Orgs.), Handbook of behavior analysis (Vol. 1, pp. 15- 18). Washington: APA Handbook in Psychology.

Skinner, B. F. (1953). Science and human behavior. Nova Iorque: Macmillan.

Skinner, B. F. (1956). What is psychotic behavior? In B. F. Skinner (Org.), Cumulative record: A selection of papers (pp. 202-219). Nova lorque: Applenton-Century-Crofts.

Skinner, B. F. (1974). About behaviorism. Nova lorque, NY: Alfred A. Knopf.
Skinner, B. F., Solomon, H., \& Lindsley, O. R. (1954). A new method for the experimental analysis of the behavior of psychotic patients. Journal of Nervous and Mental Disease, 120, 403-406.

Sousa, N. R., \& Britto, I. A. G. S. (2017). Controle do comportamento de uma pessoa com diagnóstico de esquizofrenia em comunidade evangélica. Revista Brasileira de Terapia Comportamental e Cognitiva, 19(2), 6-23. doi: 10.31505/rbtcc.v19i2.1030

Sousa, N. R., \& Britto, I. A. G. S. (2019). Drogadição: tratamento comportamental em comunidade terapêutica. Curitiba: CRV.

Vieira, G. F., \& Britto, I. A. G. S. (2019). Avaliação funcional e intervenção em respostas de dor (Tese de Doutorado, Pontifícia Universidade Católica de Goiás, Goiás). Recuperado de http://tede2.pucgoias. edu.br:8080/handle/tede/4366

Ilma Aparecida Goulart de Souza Britto, Doutora em Ciências Sociais pela Pontifíca Universidade Católica de São Paulo - PUC-SP, é

Professora Titular da Pontifícia Universidade Católica de Goiás-

PUC Goiás. Endereço para correspondência: Rua Aspília, Quadra A3 Lote 20, Residencial dos Ipês, Alphaville Flamboyant, Goiânia, GO CEP 74.884-547. Telefone: Celular: (62) 99979-0708 Fax (62) 3281-7400. Email: psyilma@terra.com.br

Roberta Maia Marcon, Doutora em Psicologia pela Pontifícia Universidade Católica de Goiás - PUC Goiás, é Professora da Pontifícia Universidade Católica de Goiás - PUC. Email:robertamarconpsi@gmail.com

Recebido em 29.mai.19 Revisado em 18.set.19 Aceito em 12.nov.19 